\title{
Colistin resistance superimposed to endemic carbapenem-resistant Klebsiella pneumoniae: a rapidly evolving problem in Italy, November 2013 to April 2014
}

\author{
M. Monaco ${ }^{1,2}$, T Giani ${ }^{2,3}$, M Raffone $^{1,4}$, F Arena $^{3}$, A Garcia-Fernandez $^{1}$, S Pollini $^{3}$, Network EuSCAPE-Italy ${ }^{5}$, H Grundmann $^{6}$, \\ A Pantosti (annalisa.pantosti@iss.it) 1 , G M Rossolini ${ }^{3,7,8}$ \\ 1. Department of Infectious, Parasitic and Immune-mediated Diseases, Istituto Superiore di Sanità, Rome, Italy \\ 2. MM and TG have equally contributed to this work \\ 3. Department of Medical Biotechnologies, University of Siena, Siena, Italy \\ 4. Federico II University Hospital, Neaples, Italy \\ 5. The network EuSCAPE-Italy participants are listed at the end of this article \\ 6. Department of Medical Microbiology, University of Groningen, University Medical Center Groningen, the Netherlands \\ 7. Department of Experimental and Clinical Medicine, University of Florence, Florence, Italy \\ 8. Clinical Microbiology and Virology Unit, Florence Careggi University Hospital, Florence, Italy
}

Citation style for this article: Monaco M, Giani T, Raffone M, Arena F, Garcia-Fernandez A, Pollini S, Network EuSCAPE-Italy, Grundmann H, Pantosti A, Rossolini GM. Colistin resistance
superimposed to endemic carbapenem-resistant Klebsiella pneumoniae: a rapidly evolving problem in Italy, November 2013 to April 2014 . Euro Surveill. 2014;19(42): pii=20939. Available online: http://www.eurosurveillance.org/ViewArticle. aspx?Articleld=20939

Article submitted on 08 October 2014 / published on 23 October 2014

Consecutive non-replicate clinical isolates $(n=191)$ of carbapenem non-susceptible Enterobacteriaceae were collected from 21 hospital laboratories across Italy from November 2013 to April 2014 as part of the European Survey on Carbapenemase-producing Enterobacteriaceae (EuSCAPE) project. Klebsiella pneumoniae carbapenemase-producing $K$. pneumoniae (KPC-KP) represented 178 (93\%) isolates with 76 (43\%) respectively resistant to colistin, a key drug for treating carbapenamase-producing Enterobacteriaceae. KPC-KP colistin-resistant isolates were detected in all participating laboratories. This underscores a concerning evolution of colistin resistance in a setting of high KPC-KP endemicity.

We report the widespread and rapid dissemination of resistance against colistin, a key drug for treatment of carbapenamase-producing Enterobacteriaceae, among Klebsiella pneumoniae carbapenemase (KPC)producing $K$. pneumoniae (KPC-KP) in Italy. As part of the European Survey on Carbapenemase-producing Enterobacteriaceae (EuSCAPE) project, consecutive non-replicate clinical isolates of carbapenem non-susceptible (resistant or intermediate) Enterobacteriaceae $(n=191)$ were collected from 21 Italian hospital laboratories between November 2013 and April 2014. Most isolates 178 (93\%) were KPC-KP, with 76 (43\%) respectively resistant to colistin. This report details the findings and discusses potential implications for infection control.

\section{Background}

Carbapenem-resistant Enterobacteriaceae (CRE) emerged in recent years as one of the most challenging group of antibiotic-resistant pathogens. Related mortality rates are high due to limited treatment options, and some strains have the potential for rapid dissemination in healthcare settings $[1,2]$. In Europe, CRE have been reported from virtually all countries, but in some countries, namely Greece and Italy, they have spread rapidly and are presently endemic in many hospitals [3,4]. Resistance to carbapenems in Enterbacteriaceae is largely due to production of enzymes (carbapenemases) inactivating these antibiotics, hence the definition of carbapenemase-producing Enterobacteriaceae (CPE).

In Italy, the dramatic increase of carbapenem-resistant Klebsiella pneumoniae has been documented by the European Antimicrobial Resistance Surveillance Network (EARS-Net) which showed that the percentage of invasive isolates of carbapenem-resistant $K$. pneumoniae, that was until 2009 lower than one to $2 \%$, increased to $15 \%$ in 2010 to reach 35\% in 2013 ([5] and unpublished data). Data provided by Micronet (http:// www.simi.iss.it/micronet.htm), a sentinel epidemiological surveillance network based on computerised daily collection of microbiological data from the laboratory information systems of 27 laboratories nationwide, confirmed the increase in the percentage of carbapenem-resistant $K$. pneumoniae in samples from different anatomical sites, including lower respiratory secretions and urine [6]. In addition, analysis of resistance determinants and clonality, revealed that the Italian CRE epidemic was mostly sustained by KPC-KP of clonal complex 258 , with only a minority of different clones and resistance mechanisms [7].

Polymyxins (colistin and polymyxin B), together with tigecycline and gentamicin, are among the few agents 
Carbapenemase determinants detected in the confirmed carbapenem non-susceptible isolates collected as part of the EuSCAPE survey, Italy, November 2013-April 2014 ( $\mathrm{n}=191)$

\begin{tabular}{|c|c|c|c|c|c|}
\hline \multirow{2}{*}{ Species } & \multicolumn{5}{|c|}{ Number of isolates per type of carbapenemase } \\
\hline & $b l a_{\mathrm{KPC}}$ & $b l a_{\mathrm{vIM}}$ & bla $_{\mathrm{NDM}}$ & $b / a_{\mathrm{OXA}-48}$ & None detected \\
\hline Klebsiella pneumoniae & $178^{\mathrm{a}}$ & 3 & 1 & 1 & 4 \\
\hline Escherichia colib & 3 & 1 & - & - & - \\
\hline
\end{tabular}

EUSCAPE: European Survey on Carbapenemase-producing Enterobacteriaceae.

K. pneumoniae carbapenemase-producing K. pneumoniae (KPC-KP) were reported from all peripheral laboratories.

a Detected from all the 21 peripheral laboratories.

b The four carbapenemase-producing $E$. coli isolates were from different peripheral laboratories.

that retain activity against KPC-KP, and are key components of the combination antimicrobial regimens that are recommended for treatment of these pathogens $[8,9]$. Therefore, the emergence of resistance to these last line drugs among KPC-KP is important to monitor.

\section{Implementation of European Survey on Carbapenemase-producing Enterobacteriaceae in Italy}

EuSCAPE is funded by the European Centre for Disease Prevention and Control (ECDC) and coordinated by the Department of Medical Microbiology of the University Medical Center Groningen in the Netherlands. This initiative aims to foster active surveillance of CPE through improving the diagnostic capacity of microbiological laboratories in Europe [10]. A crucial part of EuSCAPE consisted of a structured survey that between November 2013 and April 2014 involved hospital laboratories from 35 countries across Europe. In each participating country the National Expert Laboratory (NEL) collected and characterised clinical isolates of suspected carbapenem non-susceptible $K$. pneumoniae or Escherichia coli obtained from a sentinel network of peripheral laboratories (PLs). Each PL was asked to collect the first 10 consecutive non-replicate isolates of suspected carbapenem non-susceptible $K$. pneumoniae or $E$. coli obtained fxrom clinical samples (blood, lower respiratory tract secretions, urine, puncture fluids and wound secretions) and to provide also relevant demographic and clinical data (age, sex, location of patient in hospital, previous hospital admission in the last six months, previous stay or travel abroad within the last six months).

In Italy, a total of 21 PLs that served 45 hospitals or outpatients clinics distributed across the country participated in the survey. PLs identified suspected carbapenem non-susceptible $K$. pneumoniae or $E$. coli by automated systems Vitek 2 (bioMérieux, Marcy l'Etoile, France) or Phoenix (Becton Dickinson Diagnostic Systems, Sparks, MD, USA). Subsequently these isolates were sent to the NEL in Rome, who in collaboration with the NEL in Siena, performed confirmation and further characterisation. NELs confirmed species identification by matrix-assisted laser desorption/ionization time-of-flight (MALDI-TOF) mass spectrometry (Vitek MS, bioMérieux), and carried out susceptibility testing against carbapenems and other antimicrobial agents by reference broth microdilution [11] using commercial microtitre plates (Alere Technologies, GmbH, Jena, Germany) and manually prepared plates for colistin testing. Results were interpreted according to the European Committee on Antimicrobial Susceptibility Testing (EUCAST) clinical breakpoints [12]. The presence of carbapenemase genes of the bla $a_{K P C}, b l a_{N D M}$, bla $a_{V I M}$, and $b a_{O X A-48}$ types was investigated by polymerase chain reaction (PCR) using the protocol recommended by EuSCAPE (available upon request from the EuSCAPE Coordinator, Prof. Hajo Grundmann).

\section{Results of the survey}

A total of 197 suspected carbapenem non-susceptible $K$. pneumoniae or $E$. coli isolates were collected by the PLs in the study period. Of these, $187 \mathrm{~K}$. pneumoniae and four $E$. coli were confirmed as non-susceptible to at least one carbapenem antibiotic (imipenem, meropenem or ertapenem). The bla $a_{K P C}$ determinant was found to be the most prevalent among carbapenem non-susceptible isolates, being detected in 178 $K$. pneumoniae and in three $E$. coli, while other carbapenemase genes were infrequently found (Table).

KPC-KP were obtained from urine (67 isolates), blood (61 isolates), lower respiratory tract (21 isolates), wound secretions (10 isolates), and other specimens (19 isolates). Patients with KPC-KP had a median age of 72 years (range: 16-94 years); 106 (60\%) were males and 72 (40\%) were females. Of these patients, 41(23\%) had KPC-KP isolates detected while in intensive care unit (ICU), 127 (71\%) were found while in a medical or surgical ward, and $10(6 \%)$ were outpatients or patients seen at the emergency department. Another hospital admission in the previous six months was reported for $96(64 \%)$ of patients for whom the information was available $(n=150)$. Travelling abroad during the last six months was reported for only $3(3 \%)$ of the patients for whom the information was available $(n=111)$. Thus, $97 \%(108 / 111)$ of KPC-KP infections are endemic cases. 


\section{FIGURE}

Distribution of peripheral laboratories reporting KPCproducing K. pneumoniae isolates in the EuSCAPE Italian survey, Italy, November 2013-April 2014 ( $\mathrm{n}=191$ isolates)

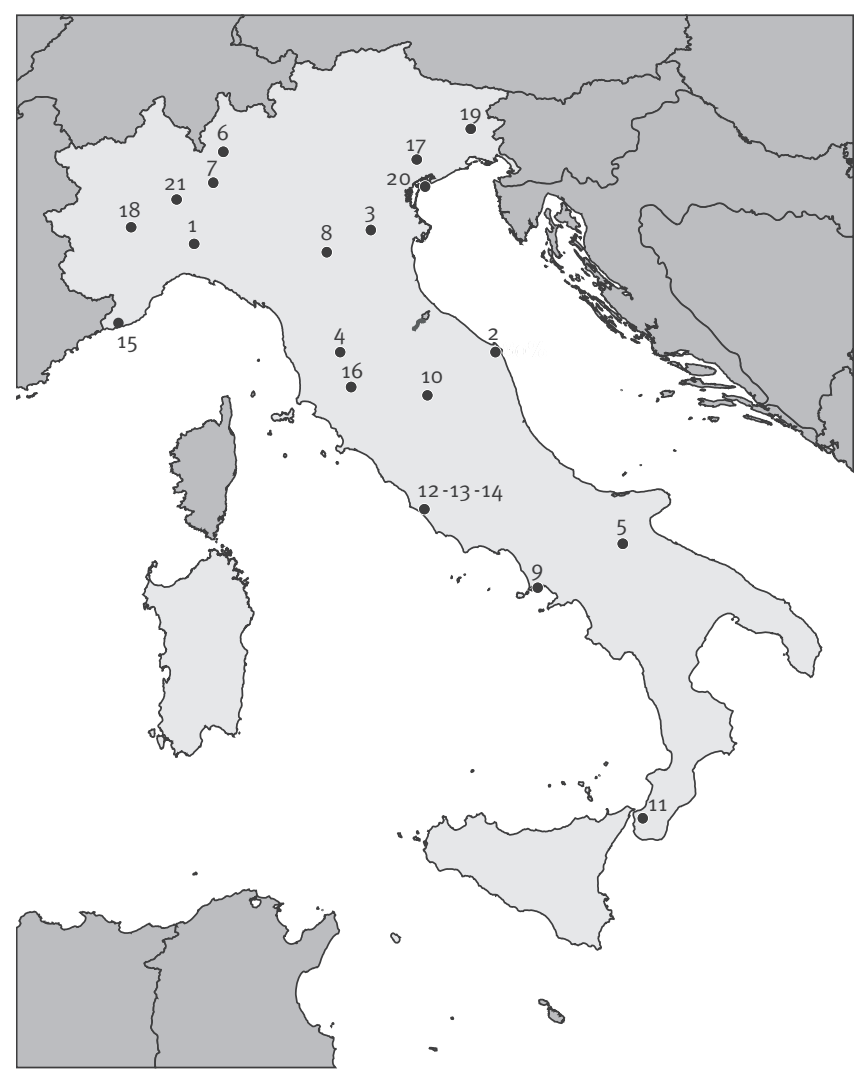

EUSCAPE: European Survey on Carbapenemase-producing Enterobacteriaceae; KPC: Klebsiella pneumoniae carbapenemase; KPC-KP: KPC-producing K. pneumoniae.

The peripheral laboratories are numbered on the map according to alphabetical order.

Proportions of colistin-resistant isolates among KPC-KP per peripheral laboratory:. 1, Alessandria: 1/10; 2, Ancona: 8/10; 3, Ferrara: 1/4; 4, Florence: 5/10; 5, Foggia: 4/10; 6, Lecco: 2/9; 7, Milan: $1 / 10 ; 8$, Modena: 3/7; 9, Neaples: 3/8; 10, Perugia: 5/10; 11, Reggio Calabria: 4/10; 12 , Rome: 4/9; 13, Rome: 2/4; 14, Rome: 6/7; 15, San Remo: 4/8; 16, Siena: 6/8; 17, Treviso: $1 / 7 ; 18$, Turin: 5/9; 19 , Udine: $2 / 8 ; 20$, Venice: $8 / 10 ; 21$, Vercelli: $1 / 10$.

Antimicrobial susceptibility data for the 178 KPC-KP isolates revealed that $76(43 \%)$ were resistant to colistin, $11(6 \%)$ resistant or intermediate to tigecycline, 29 $(16 \%)$ resistant or intermediate to gentamicin, and 146 $(82 \%)$ resistant or intermediate to trimethoprim-sulfamethoxazole (SXT). Two isolates (1\%) were resistant or intermediate to all four antibiotics. Colistin-resistant KPC-KP isolates were detected from all PLs, although at variable percentages (Figure).

\section{Discussion and conclusions}

Although most recent data from April 2014 to date are not available at this time, the results of this survey confirmed the widespread endemicity of KPC-KP in Italian healthcare facilities, and their predominant role among CPE. Infections with KPC-KP affect mostly older patients hospitalised in medical or surgical wards with a known history of previous hospital admission in the country. The results of this present study also reveal a concerning percentage of resistance to colistin, which is a matter of major concern given the dearth of treatment options against CPE.

In Italy, the emergence of colistin-resistant KPC-KP has been reported since 2010 [13] and, in the first Italian nationwide cross-sectional survey on CRE, carried out in mid-2011, the overall percentage of colistin resistance among KPC-KP was found to be $22.4 \%$, with colistin-resistant isolates reported from 13 of 25 participating hospital laboratories [7]. In the EuSCAPE study, the colistin resistance percentage found among KPC-KP was almost double, and colistin-resistant KPC-KP isolates were detected from all 21 PLs in the study. We did not have information to derive the total number of affected hospitals among the 45 served by the $21 \mathrm{PLs}$, however the PLs were distributed all across the country. A similar situation of nationwide dissemination of colistin-resistant KPC-KP has not yet been reported in other settings of high KPC-KP endemicity [14].

According to data available from the European Surveillance of Antimicrobial Consumption Network (ESAC-NET) database [15], consumption of polymixins in the hospital sector in Italy increased from 0.0017 to 0.0194 Defined Daily Dose (DDD) per 1,000 inhabitants per day in the period from 2007 to 2012. This 10 -fold increase reflects the increasing dissemination of multidrug-resistant Gram-negative infections for which colistin remains one of the few therapeutic options and most likely contributed to selection of colistin-resistant strains among KPC-KP.

To control the spread of KPC-KP in Italy, in February 2013 the Ministry of Health issued a circular letter [16] asking the Italian regions to report all cases of bloodstream infections due to CPE of the species $K$. pneumoniae or $E$. coli and recommending control measures to limit the spread in healthcare settings. These control measures consist of: (i) active screening of selected patient groups including patients who have been in contact with CPE-colonised or infected patients, and patients coming from countries with high CPE endemicity and, if feasible, patients admitted to ICU or other high-risk wards and patients with a history of previous hospitalisation; (ii) isolation or cohorting of infected/ colonised patients, separate cohort nursing care, and implementation of contact precautions, according to the recommendations issued at national and international level [17-20].

These measures require huge efforts and resources in an endemic situation like the one highlighted in this study, since patients with KPC-KP infection or colonisation are not confined to ICUs, but can be found in normal hospital wards. It seems therefore urgent to develop and implement a national plan for the prevention and control of CPE infections in Italy that includes 
an extensive surveillance system and more comprehensive guidelines on infection control measures. Sufficient resources should be allocated to contain the further dissemination of CPE in healthcare institutions.

\section{Acknowledgements}

We thank Alessandra Carattoli for helpful discussion and support to this study.

Funding: This study is part of the European Survey on Carbapenemase-Producing Enterobacteriaceae (EUSCAPE) project coordinated by the University Medical Center Groningen and funded by ECDC through a specific framework contract (ECDC/2012/055) following an open call for tender (0J/25/04/2012-PROC/2012/036). NEL in Rome was supported in part by a grant from the Italian Ministry of Health (CCM 2013 "Sorveglianza di laboratorio di infezioni batteriche da patogeni antibiotico-resistenti sottoposti a sorveglianza europea). NEL in Siena was partially supported by a research grant from EvoTAR (no. HEALTH-F3-2011-2011-282004) to G.M.R.

\section{Conflict of interest}

MM, MR, AGF, HG and AP have nothing to declare; GMR has received research grants from Pfizer, Astra-Zeneca, Cubist, Angelini, Becton-Dickinson, bioMérieux, Biotest, VenatoRx, has served as consultant for Pfizer, Astra-Zeneca, Cubist, Angelini, Menarini, Achaogen, Rempex, Durata, Medivir, Biotest, and has served in the Speaker's Bureau for Pfizer, Astra-Zeneca, Novartis, Angelini, Curetis, Biotest and Basilea. TG has served in the Speaker's Bureau for bioMérieux.

\section{Author contributions}

$M M, T G, A P$ and GMR contributed to the design of the study, to draft and finalise the manuscript; TG, SP, FA performed the phenotypic characterisation of the isolates; $M M, M R$, AGF performed the detection of the carbapenemase genes by PCR; MM, MR, AGF, TG, SP, FA entered and analysed data; HG planned and coordinated the EuSCAPE study and revised the final manuscript; PLs provided isolates and clinical and demographic data of patients.

\section{Members of the Network EuSCAPE-Italy}

A. Barbaro, Ospedali Riuniti Melacrino-Morelli, Reggio Calabria; L. Campion, Ospedale S. Maria di Ca' Foncello, Treviso; M. R. Catania, Azienda Ospedaliera Universitaria Federico II, Napoli; A. De Bernochi, Ospedale San Giovanni Bosco, Torino; A. M. Di Taranto, Azienda Mista OspedalieraUniversitaria, Foggia; P. A. Dusi, Ospedale di Sanremo, Sanremo (IM); S. Grandesso, Ospedale Dell' Angelo, Mestre (VE); F. Luzzaro, Ospedale A. Manzoni, Lecco; E. Manso, Ospedale Torrette Umberto I, Ancona; M. Meledandri, Azienda Ospedaliera San Filippo Neri, Rome; A. Mencacci, Ospedale Santa Maria della Misericordia, Perugia; F. Milano, Ospedale Sant'Andrea, Vercelli; G. Parisi, Azienda Ospedaliera San Camillo-Forlanini, Rome; P. Pecile, Azienda Ospedaliero-Universitaria Careggi, Firenze; A. Restelli, Fondazione IRCCS Cà Granda Ospedale Maggiore Policlinico, Milano; A. Rocchetti, Azienda Ospedaliera Nazionale Santi Antonio e Biagio e C. Arrigo, Alessandria; M. R. Rossi, Ospedale Universitario Sant' Anna di Cona, Ferrara; I. Santino, Azienda Policlinico S. Andrea,Rome; M. Sarti, Nuovo Ospedale Civile S. Agostino-Estense di Baggiovara,
Modena; A. Sartor, Azienda Ospedaliero-Universitaria Santa Maria della Misericordia, Udine.

\section{References}

1. Tzouvelekis LS, Markogiannakis A, Psichogiou M, Tassios PT, Daikos GL. Carbapenemases in Klebsiella pneumoniae and other Enterobacteriaceae: an evolving crisis of global dimensions. Clin Microbiol Rev. 2012;25(4):682-707. http:// dx.doi.org/10.1128/CMR.05035-11

2. Nordmann P, Naas T, Poirel L. Global spread of carbapenemase-producing Enterobacteriaceae. Emerg Infect Dis. 2011;17(10):1791-8. http://dx.doi.org/10.3201/ eid1710.110655

3. Glasner C, Albiger B, Buist G, Tambić Andrašević A, Canton R, Carmeli Y, et al. Carbapenemase-producing Enterobacteriaceae in Europe: a survey among national experts from 39 countries, February 2013. Euro Surveill. 2013;18(28): pii=20525.

4. Cantón R, Akóva M, Carmeli Y, Giske CG, Glupczynski $Y$, Gniadkowski M, et al. Rapid evolution and spread of carbapenemases among Enterobacteriaceae in Europe. Clin Microbiol Infect. 2012;18(5):413-31. http://dx.doi. org/10.1111/j.1469-0691.2012.03821.x

5. European Antimicrobial Resistance Surveillance Network (EARS-Net). Stockholm: ECDC. [Accessed 21 Oct 2014]. Available from: http://www.ecdc.europa.eu/en/activities/ surveillance/EARS-Net/Pages/index.aspx

6. Sisto A, D’ Ancona F, Meledandri M, Pantosti A, Rossolini GM, Raglio A, et al. Carbapenem non-susceptible Klebsiella pneumoniae from Micronet network hospitals, Italy, 2009 to 2012. Euro Surveill. 2012;17(33): $\mathrm{pii}=20247$.

7. Giani T, Pini B, Arena F, Conte V, Bracco S, Migliavacca R, et al. Epidemic diffusion of KPC carbapenemase-producing Klebsiella pneumoniae in Italy: results of the first countrywide survey, 15 May to 30 June 2011. Euro Surveill. 2013;18(22): $\mathrm{pii}=20489$.

8. Petrosillo N, Giannella M, Lewis R, Viale P. Treatment of carbapenem-resistant Klebsiella pneumoniae: the state of the art. Expert Rev Anti Infect Ther. 2013;11(2):159-77. http:// dx.doi.org/10.1586/eri.12.162

9. Tzouvelekis LS, Markogiannakis A, Piperaki E, Souli M, Daikos $\mathrm{GL}$. Treating infections caused by carbapenemase-producing Enterobacteriaceae. Clin Microbiol Infect. 2014;20(9):862-72. http://dx.doi.org/10.1111/1469-0691.12697

10. European Centre for Disease Prevention and Control (ECDC). Carbapenemase-producing bacteria in Europe: interim results from the European Survey on carbapenemase-producing Enterobacteriaceae (EuSCAPE) project. Stockholm: ECDC; 2013.

11. Clinical and Laboratory Standards Institute (CLSI). Methods for dilution antimicrobial susceptibility tests for bacteria that grow aerobically; approved 255 Standard. Eighth Edition. Wayne, PA: CLSI; document M7-A9.

12. European Committee on Antimicrobial Susceptibility Testing (EUCAST). Clinical breakpoints, Växjö: EUCAST. [Accessed 21 Oct 2014]. Available from: http://www.eucast.org/ clinical_breakpoints/

13. Mezzatesta ML, Gona F, Caio C, Petrolito V, Sciortino D, Sciacca $\mathrm{A}$, et al. Outbreak of KPC-3-producing, and colistin-resistant, Klebsiella pneumoniae infections in two Sicilian hospitals. Clin Microbiol Infect. 2011;17(9):1444-7.

14. Ah YM, Kim AJ. Lee JY. Colistin resistance in Klebsiella pneumoniae. Int J Antimicrob Agents. 2014;44(1):8-15. http:// dx.doi.org/10.1016/j.ijantimicag.2014.02.016

15. European Centre for Disease Prevention and Control (ECDC). Consumption of antimicrobials of Antibacterials For Systemic Use (ATC group lo1) in the community (primary care sector) in Europe, reporting year 2012. Stockholm: ECDC. [Accessed 21 Oct 2014]. Available from: http://www.ecdc.europa.eu/en/ healthtopics/antimicrobial_resistance/esac-net-database/ Pages/Antimicrobial-consumption-rates-by-country.aspx

16. Ministero della Salute. Circolare 'Sorveglianza e controllo delle infezioni da batteri produttori di carbapenemasi (CPE)'. [Circular letter 'Surveillance and control of infections due to carbapenemase-producing bacteria (CPE)]. Rome: Ministero della Salute; Feb 2014. Italian. [Accessed 21 Oct 2014]. Available from: http://www.trovanorme.salute.gov.it/norme/ renderNormsanPdf?anno $=0 \& \operatorname{codLeg}=45499 \&$ parte $=1 \% 20$ \&serie $=$

17. Grundmann H, Livermore DM, Giske CG, Canton R, Rossolini GM, Campos J, et al. Carbapenem-non-susceptible Enterobacteriaceae in Europe: conclusions from a meeting of national experts. Euro Surveill. 2010;15(46):pii=19711.

18. Schwaber MJ, Lev B, Israeli A, Solter E, Smollan G, Rubinovitch $\mathrm{B}$, et al. Containment of a country-wide outbreak 
of carbapenem-resistant Klebsiella pneumoniae in Israeli hospitals via a nationally implemented intervention. Clin Infect Dis. 2011;52(7):848-55. http://dx.doi.org/10.1093/cid/ciro25

19. Akova M, Daikos GL, Tzouvelekis L, Carmeli Y.

Interventional strategies and current clinical experience with carbapenemase-producing Gram-negative bacteria. Clin Microbiol Infect. 2012;18(5):439-48. http://dx.doi. org/10.1111/j.1469-0691.2012.03823.X

20. Tacconelli E, Cataldo MA, Dancer SJ, De Angelis G, Falcone $M$, Frank U, et al. ESCMID guidelines for the management of the infection control measures to reduce transmission of multidrug-resistant Gram-negative bacteria in hospitalized patients. Clin Microbiol Infect. 2014;20(Suppl 1):1-55. http:// dx.doi.org/10.1111/1469-0691.12427 